\title{
Revisão integrativa: distúrbios motores e o desenvolvimento da linguagem expressiva
}

\section{no Autismo}

\author{
Integrative review: motor disorders and expressive language development in Autism \\ Revisión integrativa: trastornos motores y desarrollo del lenguaje expresivo en el Autismo
}

Recebido: 21/12/2021 | Revisado: 28/12/2021 | Aceito: 30/12/2021 | Publicado: 07/01/2022

Leonel Francisco de Oliveira Freire

ORCID: https://orcid.org/0000-0002-8734-5292

Universidade do Estado do Rio Grande do Norte, Brasil

E-mail: leonelloliveira93@gmail.com

Danielle Christina Lino Leal

ORCID: https://orcid.org/0000-0002-1048-3435

Universidade do Estado do Rio Grande do Norte, Brasil

E-mail: danielleleal_@hotmail.com

Maria Aparecida Cursino

ORCID: https://orcid.org/0000-0001-9312-6056

Universidade Estadual de Campinas, Brasil

E-mail: ma.cursino@gmail.com

Anne Itamara Benigna Evangelista Aires

ORCID: https://orcid.org/0000-0002-9715-1088

Universidade do Estado do Rio Grande do Norte, Brasil

E-mail: anneaires@alu.uern.br

Marcos Daniel Oliveira e Silva

ORCID: https://orcid.org/0000-0003-2254-9398

Universidade do Estado do Rio Grande do Norte, Brasil

E-mail: marcosdaniel@alu.uern.br

Matheus da Silva Regis

ORCID: https://orcid.org/0000-0001-9402-6030

Universidade do Estado do Rio Grande do Norte, Brasil

E-mail: matheussregis@gmail.com

Marielen Ghedin da Silva

ORCID: https://orcid.org/0000-0001-8970-8563

Universidade do Extremo Sul Catarinense, Brasil E-mail: ghedin.nutri@gmail.com

Maria das Dores de Holanda Monteiro

ORCID: https://orcid.org/0000-0002-0511-5507 Instituto Federal de Educação, Ciência e Tecnologia do Ceará, Brasil

E-mail: dasdoresholandanutricionista@gmail.com

Raimunda Gerlane Lima Maia

ORCID: https://orcid.org/0000-0002-4511-5798

Instituto Federal de Educação, Ciência e Tecnologia do Ceará, Brasil

E-mail: gerlannemaia28@gmail.com

Stheshy Vieira e Souza

ORCID: https://orcid.org/0000-0001-6868-7249 Universidade do Estado do Rio Grande do Norte, Brasil

E-mail: stheshyvieira@alu.uern.br

Isabela Pinheiro Cavalcante Lima

ORCID: https://orcid.org/0000-0002-7681-9675

Universidade do Estado do Rio Grande do Norte, Brasil

E-mail: belapcl@yahoo.com.br

\section{Resumo}

O Transtorno do Espectro do Autismo (TEA) consiste em um grupo de alterações do desenvolvimento neurológico e acomete, em nível global, cerca de 1 a cada 160 crianças. Os distúrbios motores são encontrados em até $79 \%$ dos indivíduos com autismo, e estão correlacionadas ao desempenho da linguagem e o comportamento social. Este estudo buscou responder a seguinte questão: As habilidades motoras podem estar correlacionadas ao desenvolvimento da linguagem expressiva no autismo? A pesquisa foi realizada no nas bases de dados Scielo, Pubmed, Lilacs, Scopus e Web Of Science. Foram utilizados termos em inglês, padronizados e extraídos dos Descritores em Ciências da Saúde (DECS) e do Medical Subject Headings (MeSH). Foram incluídos artigos originais, publicados em periódicos nacionais e internacionais nos anos de 2015 a 2020, nos idiomas inglês e português. A pesquisa resultou em 12.822 artigos, sendo incluídos 7 artigos após a seleção. Os caminhos percorridos até o desenvolvimento e a manifestação dos 
déficits motores e da linguagem no autismo ainda constituem uma lacuna para a ciência, no entanto, a literatura científica explorada permite estabelecer uma potencial relação de interferência bilateral entre esses fatores, permitindo amplificar o universo de investigações na abordagem motora destes déficits.

Palavras-chave: Autismo; Desordem autista; Habilidades motoras; Comportamento alimentar.

\begin{abstract}
Autism Spectrum Disorder (ASD) consists of a group of neurological development disorders and affects, globally, approximately 1 in every 160 children. Motor disorders are found in up to $79 \%$ of individuals with autism, and are correlated with language performance and social behavior. This study sought to answer the following question: Can motor skills be correlated with the development of expressive language in autism? The search was carried out in the Scielo, Pubmed, Lilacs, Scopus and Web Of Science databases. Terms in English, standardized and extracted from the Health Sciences Descriptors (DECS) and the Medical Subject Headings (MeSH) were used. Original articles published in national and international journals from 2015 to 2020, in English and Portuguese, were included. The search resulted in 12,822 articles, including 7 articles after selection. The paths taken to the development and manifestation of motor and language deficits in autism still constitute a gap for science, however, the scientific literature explored allows for the establishment of a potential bilateral interference relationship between these factors, allowing the expansion of the universe of investigations. in the motor approach to these deficits.
\end{abstract}

Keywords: Autism; Autistic disorder; Motor skills; Feeding behavior.

\title{
Resumen
}

El trastorno del espectro autista (TEA) consiste en un grupo de trastornos del desarrollo neurológico y afecta, a nivel mundial, aproximadamente a 1 de cada 160 niños. Los trastornos motores se encuentran en hasta el $79 \%$ de las personas con autismo y se correlacionan con el rendimiento del lenguaje y el comportamiento social. Este estudio buscó responder a la siguiente pregunta: ¿Se pueden correlacionar las habilidades motoras con el desarrollo del lenguaje expresivo en el autismo? La búsqueda se realizó en las bases de datos Scielo, Pubmed, Lilacs, Scopus y Web Of Science. Se utilizaron términos en inglés, estandarizados y extraídos de los Descriptores de Ciencias de la Salud (DECS) y los Encabezamientos de Materia Médica (MeSH). Se incluyeron artículos originales publicados en revistas nacionales e internacionales de 2015 a 2020, en inglés y portugués. La búsqueda dio como resultado 12,822 artículos, incluidos 7 artículos después de la selección. Los caminos tomados hacia el desarrollo y manifestación de los déficits motores y del lenguaje en el autismo aún constituyen un vacío para la ciencia, sin embargo, la literatura científica explorada permite establecer una potencial relación de interferencia bilateral entre estos factores, permitiendo la expansión del universo de investigaciones. .en el enfoque motor de estos déficits.

Palabras clave: Autismo; Trastorno autista; Habilidades motoras; Comportamiento alimentario.

\section{Introdução}

O Transtorno do Espectro do Autismo (TEA) consiste em um grupo de alterações do desenvolvimento neurológico e acomete, em nível global, cerca de 1 a cada 160 crianças. Sua etiologia complexa envolve aspectos genéticos e ambientais como potenciais fatores desencadeantes. $\mathrm{Na}$ ausência de tratamento específico, o diagnóstico e a intervenção terapêutica precoce constituem as principais alternativas no manejo da condição (Anzulewicz et al., 2016).

Déficits no comportamento social, na comunicação, bem como a presença de comportamentos repetitivos e restritivos constituem as principais manifestações do TEA (DeMand et al., 2015). Além disso, distúrbios motores são encontrados em até $79 \%$ dos indivíduos com autismo, e é observado como um dos primeiros sinais a surgir ainda na infância. Essa condição impacta o desenvolvimento e pode comprometer a qualidade de vida pela imposição de limitações no relacionamento com o meio, gerando maior dependência nas relações sociais e no desenvolvimento da linguagem (Thompson et al., 2017)

O desenvolvimento das habilidades motoras é resultante de uma série de conexões cognitivas multicamadas. Esse processo pode ser analisado como indicador para o desfecho do desenvolvimento humano. Apesar da acentuada variabilidade das manifestações do TEA, é sugerida a existência de falhas nesse mecanismo como decorrência de uma atividade neural atípica (Ashley et al., 2020; Cavallo et al., 2018; Liu et al., 2016).

O desenvolvimento motor típico consiste no controle de reflexos primitivos. No autismo, esse processo é alterado, culminando na dificuldade de execução do movimento de forma hábil. Alterações motoras nessa fase do desenvolvimento humano podem ser indicadores dos principais distúrbios característicos do autismo (Chinello et al., 2018). Além disso, a sincronia social, que consiste na capacidade de integrar e processar comandos do ambiente e de pessoas e posteriormente 
elaborar uma resposta, pode ser empreendida a partir do funcionamento motor (Fitzpatrick et al., 2017).

Diante da relevância dos eventos motores como indicador do desenvolvimento em diferentes áreas do comportamento humano, bem como da importância da identificação e intervenção precoce desses distúrbios em crianças autistas, este estudo visa integrar esses fatores no manejo do autismo através de uma revisão integrativa da literatura científica, buscando responder a seguinte questão: As habilidades motoras podem estar correlacionadas ao sincronismo verbal e habilidades orais no autismo?

\section{Metodologia}

Este estudo trata-se de uma revisão integrativa da literatura. A pesquisa foi realizada no mês de abril de 2020 nas bases de dados Scielo, Pubmed, Lilacs, Scopus e Web Of Science. Utilizaram-se os termos em inglês: "autism AND motor AND dysfunction AND oral"; "autism AND motor skill AND oral"; "autism AND motor skill AND oral AND food"; "autistic disorder AND feeding behavior"; "autistic disorder AND feeding behavior AND motor skill”; "autism AND motor skill AND oral AND speaks"; "autistic disorder AND feeding behavior AND speaks"; "autistic disorder AND feeding behavior AND motor skill AND speaks; "autism AND motor skill AND oral AND language"; "autistic disorder AND feeding behavior AND language"; "autistic disorder AND feeding behavior AND motor skill AND language"; "autism AND motor skills AND food"; "autism AND motor skills AND language"; "autism AND motor skills AND eating"; "autism AND motor skills AND food consumption"; "autism AND feeding and eating disorders"; "autism AND motor skill AND oral AND verbal behavior"; "autistic disorder AND feeding behavior AND verbal behavior" e "autistic disorder AND feeding behavior AND motor skill AND verbal behavior", padronizados e extraídos dos Descritores em Ciências da Saúde (DECS) e do Medical Subject Headings (MeSH).

Constituíram-se como critérios de inclusão: Artigos originais, publicados em periódicos nacionais e internacionais nos anos de 2015 a 2020, nos idiomas inglês e português. Artigos relacionados ao autismo, alterações motoras, comportamento social e alimentar e a linguagem. Optou-se por estudos cujas metodologias incluíssem a aplicação de ferramentas validadas para investigação da presença de distúrbios motores e suas implicações no autismo. A pesquisa resultou em 12.822 artigos. Foram eliminados 22 artigos duplicados, e após a etapa de seleção por títulos restaram 177 artigos. Após a aplicação dos critérios de inclusão e exclusão, a amostra final foi constituída por 7 artigos. A Figura 1 traz o fluxograma ilustrado da pesquisa. 
Figura 1: Fluxograma de seleção de artigos.

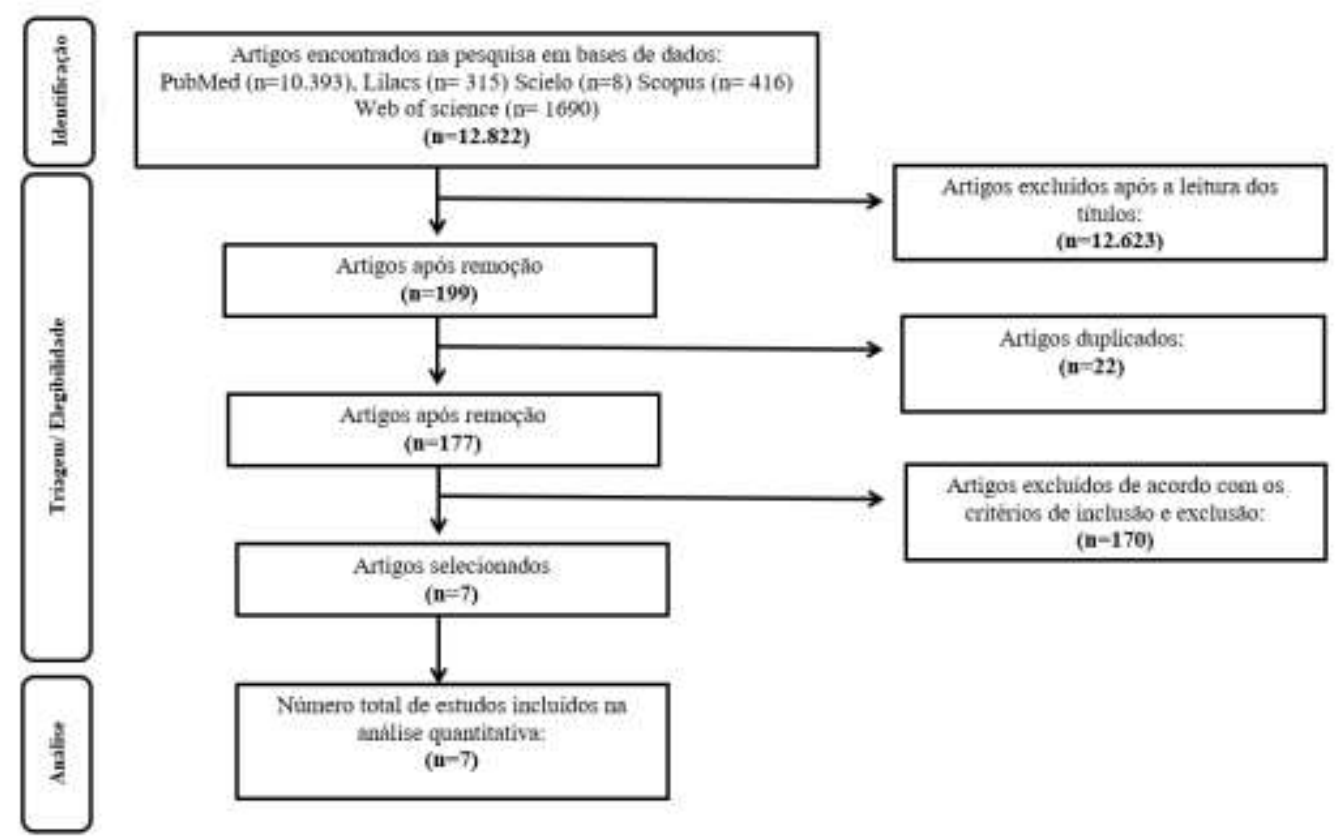

Fonte: Autores.

\section{Resultados}

Esta revisão integrativa da literatura analisou 7 estudos, selecionados de acordo com os critérios de inclusão e exclusão. Somadas, a população investigada nesses estudos corresponde a 3.634 indivíduos, em sua maioria, crianças com diagnóstico de TEA.

A análise da literatura explorada nesta revisão integrativa permitiu distribuir os principais achados em quatro categorias: Funcionamento motor e o comportamento social; Funcionamento motor e a capacidade de imitação; Funcionamento motor e a linguagem; Funcionamento motor e repercussões alimentares. Essa relação está expressa de forma esquematizada na Figura 2.

Figura 2: Variáveis relacionadas ao desempenho motor e a linguagem.

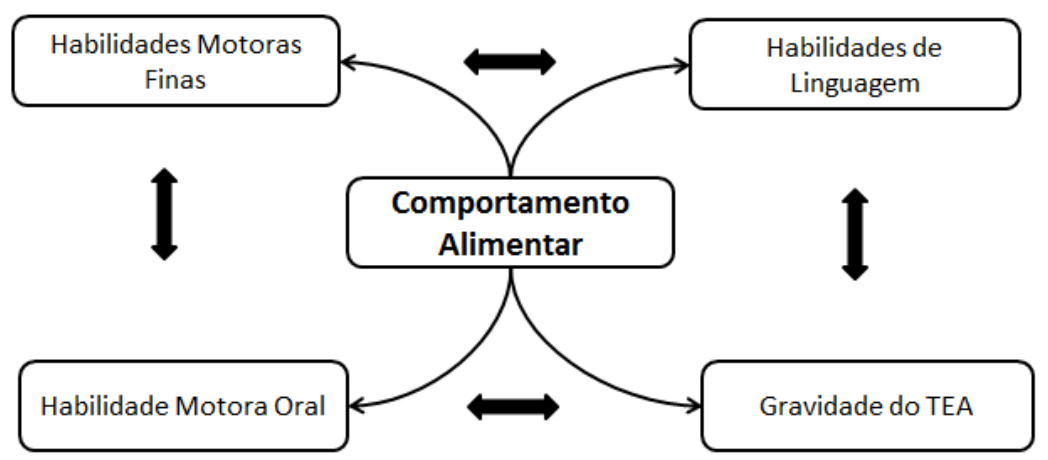

Legenda: O desenvolvimento da linguagem no autismo pode ser interpretado a partir da interação entre as habilidades motoras finas e orais, desenvolvimento da linguagem (expressiva e receptiva) e o grau do autismo. Esses aspectos conversam entre si e definem a forma como ocorre a relação com o ambiente, a construção e expressão da linguagem. Fonte: Autores. 
As avaliações da função motora, comportamento social, desenvolvimento da linguagem e comportamento alimentar, bem como dos fatores associados, foram realizadas nos estudos com a utilização de ferramentas validadas, conforme descrito na Tabela 1.

Os principais achados mostram que a observação do comportamento motor em crianças autistas, desde as primeiras manifestações no início da vida, exerce um potencial mecanismo para a previsão do desenvolvimento posterior do autismo, e o estabelecimento do grau de desenvolvimento cognitivo, da linguagem e interação social ao longo da vida.

A Tabela 1 mostra os principais pontos analisados.

Tabela 1: Resumo dos principais pontos abordados nos estudos.

\begin{tabular}{|c|c|c|c|}
\hline Autor & Objetivo do Estudo & População e Metodologia & Principais achados \\
\hline $\begin{array}{l}\text { BAL VH et al., } \\
2019\end{array}$ & $\begin{array}{l}\text { Examinar preditores de } \\
\text { desenvolvimento expressivo } \\
\text { da linguagem em pré-escolares } \\
\text { com atraso na linguagem, } \\
\text { seguidas até a idade escolar e a } \\
\text { idade adulta jovem. }\end{array}$ & $\begin{array}{l}\text { Participaram do estudo } 86 \\
\text { crianças diagnosticadas com } \\
\text { TEA. Foram realizadas } \\
\text { avaliações aos } 3 \text { anos de } \\
\text { idade e posteriormente aos } 9 \\
\text { e } 19 \text { anos. Foi aplicada a } \\
\text { Escalas de Desenvolvimento } \\
\text { Revisadas pela Merrill - } \\
\text { Palmer, Escala de Vineland e } \\
\text { Programa de Observação do } \\
\text { Diagnóstico do Autismo } \\
\text { (ADOS). }\end{array}$ & $\begin{array}{l}\text { O estudo identificou uma forte correlação entre } \\
\text { atrasos nas habilidades motoras finas e problemas } \\
\text { posteriores de linguagem. Crianças que aos } 3 \text { anos de } \\
\text { idade possuíam atrasos no motor fino expressaram } \\
\text { palavras únicas e tiveram menos ganhos na linguagem } \\
\text { expressiva ao longo do tempo. Foi estabelecido a } \\
\text { correlação entre habilidades motoras orais, motor fino } \\
\text { e desenvolvimento da linguagem. Destaca-se uma } \\
\text { relação entre o desenvolvimento motor e expressivo } \\
\text { da linguagem e aponta para atrasos motores finos } \\
\text { graves como um marcador particularmente relevante } \\
\text { na identificação de crianças com TEA com atraso na } \\
\text { linguagem que correm risco de apresentar problemas } \\
\text { de linguagem persistentes. }\end{array}$ \\
\hline $\begin{array}{l}\text { BEDFORD et al., } \\
2016\end{array}$ & $\begin{array}{l}\text { Investigar se a capacidade } \\
\text { motora e a idade do início da } \\
\text { caminhada preveem trajetórias } \\
\text { subsequentes de linguagem } \\
\text { receptiva e expressiva entre } 2 \\
\text { e } 9 \text { anos de idade em crianças } \\
\text { com TEA. }\end{array}$ & $\begin{array}{l}\text { Participaram do estudo } 209 \\
\text { crianças menores de } 37 \text { meses } \\
\text { com diagnóstico de TEA. } \\
\text { Foram analisadas aos } 2,3,5 \text { e } \\
9 \text { anos, as habilidades diárias, } \\
\text { desenvolvimento motor e } \\
\text { cognitivo. Foram utilizadas as } \\
\text { ferramentas: Escalas de } \\
\text { comportamento adaptativo de } \\
\text { Vineland (VABS-II), Escalas } \\
\text { Mullen de aprendizagem } \\
\text { precoce (MSEL) e a } \\
\text { Entrevista sobre diagnóstico } \\
\text { de autismo - revisada (ADI- } \\
\text { R). }\end{array}$ & $\begin{array}{l}\text { O estudo observou existir uma relação significativa } \\
\text { entre a caminhada e a inclinação da linguagem } \\
\text { receptiva de } 2 \text { a } 9 \text { anos. O estudo encontrou } \\
\text { evidências de uma relação entre habilidades motoras } \\
\text { precoces e posterior desenvolvimento de linguagem } \\
\text { receptiva e expressiva em crianças com autismo. }\end{array}$ \\
\hline $\begin{array}{l}\text { DADGAR et al., } \\
2017\end{array}$ & $\begin{array}{l}\text { Investigar a associação de } \\
\text { habilidades motoras e imitação } \\
\text { com habilidades iniciais de } \\
\text { comunicação social em } \\
\text { crianças com transtorno do } \\
\text { espectro autista (TEA) }\end{array}$ & $\begin{array}{l}\text { Participaram do estudo } 20 \\
\text { crianças com TEA, com idade } \\
\text { de } 3 \text { a } 5 \text { anos. As crianças } \\
\text { foram submetidas a Escala de } \\
\text { Imitação Motora (MIS), Teste } \\
\text { de desenvolvimento motor } \\
\text { bruto (TGMD-2) e Escalas } \\
\text { iniciais de comunicação } \\
\text { social (ESCS). }\end{array}$ & $\begin{array}{l}\text { Pode-se concluir que tanto a capacidade de imitação } \\
\text { quanto a função motora têm associação com as } \\
\text { habilidades sociais e de linguagem. No entanto, esses } \\
\text { resultados indicaram que pode ser benéfico para o } \\
\text { TEA visar a imitação e habilidades motoras em } \\
\text { programas de intervenção precoce. Futuramente, } \\
\text { novos estudos devem aplicar e avaliar protocolos de } \\
\text { intervenção que permitam relacionar as habilidades } \\
\text { motoras e a comunicação no TEA. }\end{array}$ \\
\hline $\begin{array}{l}\text { DALTON et al., } \\
2017\end{array}$ & $\begin{array}{l}\text { Examinar a relação entre a } \\
\text { capacidade de atenção } \\
\text { articular e a habilidade de } \\
\text { imitação oromotora em três } \\
\text { grupos de crianças com e sem } \\
\text { Transtorno do Espectro do } \\
\text { Autismo (TEA), usando } \\
\text { tarefas de imitação verbal e } \\
\text { não verbal. }\end{array}$ & 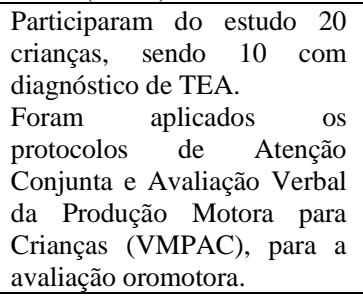 & $\begin{array}{l}\text { Em crianças verbais com TEA, a atenção conjunta e o } \\
\text { desenvolvimento motor oral podem servir como } \\
\text { componentes de um importante mecanismo de } \\
\text { acoplamento no desenvolvimento da comunicação } \\
\text { falada e no desenvolvimento posterior de habilidades } \\
\text { sócio cognitivas. }\end{array}$ \\
\hline $\begin{array}{l}\text { MCDANIEL J et } \\
\text { al., } 2018\end{array}$ & $\begin{array}{l}\text { Determinar se a atenção ao } \\
\text { falante e ao desempenho } \\
\text { motor oral prediz o tamanho } \\
\text { de vocabulário receptivo- } \\
\text { expressivo em crianças } \\
\text { pequenas com TEA. }\end{array}$ & $\begin{array}{l}\text { Participaram do estudo } 65 \\
\text { crianças com TEA. Foram } \\
\text { aplicadas as ferramentas: } \\
\text { Formulário Palavras e Gestos } \\
\text { do (MB-CDI) e Oral Motor } \\
\text { Examination. }\end{array}$ & $\begin{array}{l}\text { As habilidades motoras orais imitativas e não } \\
\text { imitativas previram a magnitude das discrepâncias de } \\
\text { tamanho do vocabulário receptivo-expressivo em } \\
\text { crianças pequenas com TEA, porém, a capacidade de } \\
\text { atenção mostrou uma relação ainda maior com o } \\
\text { vocabulário. }\end{array}$ \\
\hline $\begin{array}{l}\text { MODY et al., } \\
2017\end{array}$ & $\begin{array}{l}\text { Investigar a relação entre os } \\
\text { domínios motores a fala e a } \\
\text { interação social. }\end{array}$ & \begin{tabular}{l}
\multicolumn{3}{l}{ Participaram do estudo 1781} \\
crianças. Foi utilizada a \\
Escalas $\quad$ Mullen $\quad$ de \\
Aprendizagem Precoce e a \\
Vineland Adaptive Behavior
\end{tabular} & $\begin{array}{l}\text { O estudo demonstrou relação entre o desempenho } \\
\text { motor fino e a linguagem expressiva e receptiva, sem } \\
\text { relação significativa entre o motor grosseiro e a } \\
\text { linguagem. Os achados revelam existir uma relação } \\
\text { potencial entre o desempenho motor fino e grosseiro }\end{array}$ \\
\hline
\end{tabular}




\begin{tabular}{|c|c|c|c|}
\hline & & $\begin{array}{l}\text { Scales-I, para avaliar as } \\
\text { habilidades motoras finas e } \\
\text { grosseiras e interação social. } \\
\text { Foi utilizado também Escala } \\
\text { de Linguagem (PLS-IV e V), } \\
\text { Avaliação Clínica de } \\
\text { Fundamentos da Linguagem } \\
\text { (CELF-IV, CELF-P2), Oral e } \\
\text { Escalas de Linguagem Escrita } \\
\text { (OWLS)] e QI. }\end{array}$ & na linguagem. \\
\hline $\begin{array}{l}\text { STEVENSON et } \\
\text { al., } 2017\end{array}$ & $\begin{array}{l}\text { Explorar a relação entre } \\
\text { habilidades motoras precoces, } \\
\text { avaliadas retrospectivamente e } \\
\text { as habilidades pragmáticas } \\
\text { atuais da linguagem. }\end{array}$ & $\begin{array}{l}\text { Participaram do estudo } 49 \\
\text { crianças neurotípicas (n=20) } \\
\text { e autistas (n=29). Os } \\
\text { cuidadores das crianças } \\
\text { responderam as ferramentas } \\
\text { de avaliação da capacidade } \\
\text { linguística e a Lista de } \\
\text { Verificação da Comunicação } \\
\text { da Criança, Inventário de } \\
\text { Desenvolvimento } \\
\text { Comunicativo MacArthur, } \\
\text { Escala de medidas Autísticas } \\
\text { e a Escala de } \\
\text { Responsabilidade Social. }\end{array}$ & $\begin{array}{l}\text { As primeiras atipicidades no espectro do autismo estão } \\
\text { no domínio motor, não na comunicação social, } \\
\text { desafios ou atrasos motores podem desempenhar um } \\
\text { papel na comunicação autista, destacando o valor de } \\
\text { explorar ainda mais o papel da dispraxia na } \\
\text { comunicação autista. }\end{array}$ \\
\hline
\end{tabular}

Fonte: Autores.

\section{Discussão}

O desenvolvimento motor no início da infância constitui um aspecto importante no estabelecimento da interação e comunicação social, sendo justificado pelo acentuado aumento da interação com objetos, pessoas e o ambiente, através de gestos e vocalizações. Nessa fase, ocorre também à progressão das habilidades linguísticas que incluem a expressão e a recepção (Bradshaw et al., 2018).

As habilidades motoras envolvem uma série de conexões cognitivas multicamadas. No TEA, esse mecanismo apresenta déficits, sugerindo um funcionamento neural atípico que repercute nos diferentes distúrbios motores manifestados ainda nos primeiros meses de vida. Essas alterações podem constituir a base para a identificação dos primeiros sinais autísticos e o desenvolvimento atípico (Cavallo et al., 2018).

A supressão dos reflexos primários da infância é interpretada como um ponto importante do desenvolvimento motor. Esses reflexos são suprimidos de forma paralela a maturação do sistema nervoso, em indivíduos com desenvolvimento típico. No autismo, ocorre uma falha nesse mecanismo permitindo a permanência destes reflexos em diferentes fases do desenvolvimento, acarretando prejuízos motores (Chinello et al., 2018).

O estudo do funcionamento motor no autismo é amplo e envolve a integração entre diversas variáveis, repercutindo em diferentes aspectos do desenvolvimento, como o comportamento social, a linguagem e a alimentação. Esse estudo deve incluir também os pais, uma vez que, a identificação de trações autísticos paternos ou maternos, pode contribuir para u entendimento mais rápida das manifestações no indivíduo. Nesse contexto, é necessário que os aspectos motores sejam abordados inicialmente a partir de uma visão global, até alcançar individualmente as micro esferas do desenvolvimento.

\section{Funcionamento motor e o comportamento social}

A forma como o autista se relaciona com o ambiente permite o diálogo entre diferentes hipóteses. No entanto, o sincronismo social pode ser apontado com um termo comum nessa discussão. No TEA os distúrbios da sincronia podem ser analisados sob o aspecto cognitivo, motor e das habilidades de linguagem verbal.

Partindo do aspecto cognitivo, as crianças com TEA possuem dificuldades ao estabelecer uma conexão entre o comportamento motor das pessoas, composto por gestos e ações, e o seu próprio movimento motor, que seria uma espécie de resposta ao estímulo e estabelecimento do diálogo. A sincronia exigida nesse mecanismo, estímulo e resposta, envolve um 
complexo trabalho cognitivo relacionado à habilidade de sintetizar comandos sociais verbais, não verbais e espaciais (Fitzpatrick et al., 2017).

A interação com o ambiente é iniciada nos primeiros meses de vida a partir do desenvolvimento motor, que estimula a interação do bebê com objetos e o ambiente. Essa fase, que evolui até a caminhada, é considerada um termômetro capaz de mensurar o nível de desenvolvimento, e um marco na maturação das habilidades verbais e não verbais. No TEA esse processo sofre atenuações, ocorrendo de forma lenta, quando comparado a crianças com desenvolvimento típico (Bradshaw et al., 2018).

O comportamento social no TEA pode ser entendido a partir de dois aspectos: a demanda pessoal e a demanda externa. Quando se trata de uma resposta baseada em uma necessidade própria, a criança tende a modular com maior destreza a sua resposta motora, no entanto, quando se trata de uma ação proveniente da necessidade de sincronia com um estímulo externo, a resposta é retardada, revelando uma alteração no processamento cognitivo do diálogo (Cavallo et al., 2018)

Os déficits na responsividade sensorial em conjunto com as alterações na linguagem receptiva, distúrbios da atenção, regulação emocional e coordenação motora fina, que afeta a destreza e regulação dos movimentos, podem modular a forma como ocorre à sincronia social. Uma vez que é necessária a interação entre esses fatores para uma adequada assimilação dos comandos externos e a emissão de respostas promovendo o diálogo (Hannant et al., 2016).

É importante entender a forma como ocorre à interação entre as diferentes facetas do autismo. A análise do comportamento social permite estabelecer a relação entre a cognição, às habilidades motoras e a linguagem. $\mathrm{O}$ entendimento global dessas variáveis e a sua correlação com outros aspectos da condição, permitem o estabelecimento de uma linha de raciocínio acurada e melhor norteamento e manejo do autismo.

\section{Funcionamento motor e a capacidade de imitação}

A capacidade de imitação revela a maturação de uma ampla rede de variáveis que interferem em diversos aspectos do desenvolvimento. Esse processo permite a ascensão de cada indivíduo a partir da reprodução de fatos observados. Na cognição a imitação indica a capacidade de receber, processar e emitir respostas a diferentes estímulos. Na linguagem verbal, a capacidade de imitação demonstra o funcionamento adequado da atenção e contribui para a aquisição de novas palavras e expansão do vocabulário. Ao considerar as habilidades motoras, a imitação permite a reprodução de ações e emissão de respostas ao meio através de movimentos e gesticulações.

A imitação é um sinal precursor da cognição social que envolve, posteriormente, uma gama de conexões complexas que se desenvolvem ao longo dos primeiros anos de vida. A análise e a compreensão prévia desse fenômeno contribuem para o entendimento das implicações sociais do autismo, podendo revelar uma série de distúrbios como alterações no sistema de neurônios espelho. Paralelamente a esse processo, a manifestação de distúrbios motores pode modelar o comportamento social no TEA (Fitzpatrick et al., 2017).

A literatura científica analisada relata que a imitação estimula a linguagem, a reciprocidade social e permite compreender o grau de desenvolvimento do indivíduo. Essa aptidão é associada à práxis oral, composta por uma série de músculos da região da boca, bochechas, lábios e língua, atuantes de forma combinada na função motora acústica e linguística da fala. O movimento oromotor é responsável pela imitação verbal, reprodução de fonemas e sílabas, e a imitação não verbal, que consiste na execução de atividades orais como a movimentação dos lábios em conjunto com uma resposta visual ou gesticulação tátil (Dalton et al., 2017).

Em criança diagnosticadas com TEA, as habilidades motoras, a capacidade de imitação e o comportamento social estão inter-relacionados, indicando que pode ser benéfico o direcionamento dos programas de intervenção precoce para as habilidades de motoras e de imitação (Dadgar et al., 2017).

O desempenho da imitação no autismo pode ser analisado juntamente com o desempenho da atenção conjunta, que é a 
capacidade de administrar a atenção entre pessoas, objetos e o ambiente. Dessa forma, as crianças com maior dificuldade no estabelecimento da atenção conjunta, também apresentam desempenho oromotor inferior e distúrbios na imitação, quando comparadas a crianças com desenvolvimento típico (Dalton et al., 2017).

\section{Funcionamento motor e a linguagem}

A progressão da linguagem e das características fonológicas é associada ao desenvolvimento do comportamento motor, que inclui as habilidades motoras finas e grosseiras, e ambos estão fortemente correlacionados ao autismo e a sua progressão ao longo da vida. A construção dessa relação pode contribuir para uma melhor interpretação dos fenômenos que envolvem a verbalização, uma vez que, o mecanismo responsável pela produção da fala mostra congruência ao desempenho motor.

Ao trabalhar uma abordagem motora dos distúrbios da linguagem verbal, as habilidades motoras orais contribuem para a produção da fala. A compreensão desse processo inicia por volta dos 8 aos 10 meses de idade, e a pronúncia das primeiras palavras ocorre a partir dos 12 meses. No autismo, há um déficit na capacidade de atenção ao orador e na habilidade motora oral. As falhas na atenção ao orador prejudicam a capacidade de compreensão e aquisição de novas palavras. Já as falhas motoras orais correspondem a um déficit no planejamento motor, que inclui a fala e ações corporais, a partir da compreensão dos comandos do ambiente (Bal et al., 2019; McDaniel et al., 2018).

As primeiras alterações na linguagem e nas habilidades motoras finas surgem junto aos primeiros sinais de autismo, ainda nos meses iniciais da vida. Os atrasos da linguagem verbal se apresentam conjuntamente com os de caráter comportamental, estimando-se que até $92,4 \%$ das crianças podem sofrer esses impactos e apresentar ausência do reflexo de sucção, hipotonia neonatal e distúrbio no diálogo hipotônico, que estão relacionados ao funcionamento das estruturas mecânicas orais. Secundariamente, essas alterações se associam a distúrbios da alimentação no futuro (Parmeggiani et al., 2019).

As manifestações motoras iniciais se correlacionam ao desenvolvimento do autismo, a partir da análise do início da caminhada, do desenvolvimento da linguagem e o processamento sensorial. Partindo dessa premissa, observa-se que as crianças cujo início da caminhada ocorreu de forma tardia, também tendem a apresentar atrasos na linguagem receptiva e expressiva, sugerindo que os primeiros marcos motores possuem caráter multissensorial e integram um sistema progressivo de desenvolvimento. A visão global desses fatores revela a combinação entre a aptidão motora e as linguagens expressivas e receptivas, de forma que os déficits identificados podem ser úteis para descrever o grau de processamento sensorial(Bedford et al., 2016; Mody et al., 2017; Stevenson et al., 2017).

A literatura científica explora amplamente as correlações entre o autismo, habilidades motoras finas e grosseiras e o desempenho linguagem expressiva e receptiva. $\mathrm{O}$ agrupamento dessas características permite compreender a existência de uma relação inversa entre os traços autísticos e o desempenho da linguagem. Desse modo as crianças autistas que apresentam menores habilidades motoras orais e manuais, tendem a apresentar intensificação desses déficits no futuro e redução do desempenho na linguagem (Fulceri et al., 2019; Stevenson et al., 2017; Yang et al., 2016).

Os caminhos percorridos até o desenvolvimento e a manifestação dos déficits motores e da linguagem no autismo ainda constituem uma lacuna para a ciência. No entanto, é possível identificar a existência de uma potencial relação e interferência bilateral entre esses fatores, permitindo amplificar o universo de investigações na abordagem motora dos déficits na linguagem característicos do autismo.

\section{Conclusão}

Indivíduos com autismo tendem a apresentar um déficit significativo no sincronismo entre a cognição, habilidades motoras finas e grosseiras e o desenvolvimento linguagem verbal e não verbal. Esses fatores são envolvidos nos principais 
distúrbios da condição, se manifestando em diferentes estágios do desenvolvimento.

Os caminhos percorridos até o desenvolvimento e a manifestação dos déficits motores orais e da linguagem no autismo ainda constituem uma lacuna para a ciência. No entanto, é possível identificar a existência de uma potencial relação e interferência bilateral entre as repercussões motoras e o desempenho da linguagem, esses sinais se manifestam já nos primeiros anos de vida. Além disso, a literatura científica sugere a ampliação do universo de investigações na abordagem motora do autismo, englobando o estudo do comportamento dos pais como mecanismo norteador do diagnóstico precoce dos filhos.

Este estudo estimula a busca por uma nova abordagem para o melhor entendimento autismo, considerando as repercussões motoras como um dos pilares da avaliação do desenvolvimento na condição. Propomos a realização de novas pesquisas abordando a temática, incluindo maior aparato científico e metodológico, incluindo também, o aprofundamento e reflexão sobre a literatura científica já existente.

\section{Referências}

Anzulewicz, A., Sobota, K., \& Delafield-Butt, J. T. (2016). Toward the Autism Motor Signature: Gesture patterns during smart tablet gameplay identify children with autism. Scientific Reports, 6. https://doi.org/10.1038/srep31107.

Ashley, K., Steinfeld, M. B., Young, G. S., \& Ozonoff, S. (2020). Onset, Trajectory, and Pattern of Feeding Difficulties in Toddlers Later Diagnosed with Autism. Journal of Developmental and Behavioral Pediatrics JDBP, 41(3), 165-171. https://doi.org/10.1097/DBP.0000000000000757.

Bal, V. H., Fok, M., Lord, C., Smith, I. M., Mirenda, P., Szatmari, P., Vaillancourt, T., Volden, J., Waddell, C., Zwaigenbaum, L., Bennett, T., Duku, E., Elsabbagh, M., Georgiades, S., Ungar, W. J., \& Zaidman-Zait, A. (2019). Predictors of longer-term development of expressive language in two independent longitudinal cohorts of language-delayed preschoolers with Autism Spectrum Disorder. Journal Of Child Psychology AND PSYCHIATRY. https://doi.org/10.1111/jcpp.13117.

Bedford, R., Pickles, A., \& Lord, C. (2016). Early gross motor skills predict the subsequent development of language in children with autism spectrum disorder. Autism Research, 9(9), 993-1001. https://doi.org/10.1002/aur.1587.

Bradshaw, J., Klaiman, C., Gillespie, S., Brane, N., Lewis, M., \& Saulnier, C. (2018). Walking Ability is Associated with Social Communication Skills in Infants at High Risk for Autism Spectrum Disorder. Infancy, 23(5), 674-691. https://doi.org/10.1111/infa.12242.

Cavallo, A., Romeo, L., Ansuini, C., Podda, J., Battaglia, F., Veneselli, E., Pontil, M., \& Becchio, C. (2018). Prospective motor control obeys to idiosyncratic strategies in autism. Scientific Reports, 8(1). https://doi.org/10.1038/s41598-018-31479-2.

Chinello, A., Di Gangi, V., \& Valenza, E. (2018). Persistent primary reflexes affect motor acts: Potential implications for autism spectrum disorder. Research in Developmental Disabilities, 83, 287-295. https://doi.org/10.1016/j.ridd.2016.07.010.

Dadgar, H., Alaghband Rad, J., Soleymani, Z., Khorammi, A., McCleery, J., \& Maroufizadeh, S. (2017). The Relationship between Motor, Imitation, and Early Social Communication Skills in Children with Autism . Iranian Journal of Psychiatry, 12(4), 236-240.

Dalton, J. C., Crais, E. R., \& Velleman, S. L. (2017). Joint attention and oromotor abilities in young children with and without autism spectrum disorder. Journal of Communication Disorders, 69, 27-43. https://doi.org/10.1016/j.jcomdis.2017.06.002.

DeMand, A., Johnson, C., \& Foldes, E. (2015). Psychometric Properties of the Brief Autism Mealtime Behaviors Inventory. Journal of Autism and Developmental Disorders, 45(9), 2667-2673. https://doi.org/10.1007/s10803-015-2435-4.

Fitzpatrick, P., Romero, V., Amaral, J. L., Duncan, A., Barnard, H., Richardson, M. J., \& Schmidt, R. C. (2017). Evaluating the importance of social motor synchronization and motor skill for understanding autism. Autism Research, 10(10), 1687-1699. https://doi.org/10.1002/aur.1808.

Fulceri, F., Grossi, E., Contaldo, A., Narzisi, A., Apicella, F., Parrini, I., Tancredi, R., Calderoni, S., \& Muratori, F. (2019). Motor Skills as Moderators of Core Symptoms in Autism Spectrum Disorders: Preliminary Data From an Exploratory Analysis With Artificial Neural Networks. Frontiers in Psychology, 9. https://doi.org/10.3389/fpsyg.2018.02683.

Hannant, P., Cassidy, S., Tavassoli, T., \& Mann, F. (2016). Sensorimotor Difficulties Are Associated with the Severity of Autism Spectrum Conditions. Frontiers in Integrative Neuroscience, 10(AUGUST2016). https://doi.org/10.3389/fnint.2016.00028.

Liu, X., Liu, J., Xiong, X., Yang, T., Hou, N., Liang, X., Chen, J., Cheng, Q., \& Li, T. (2016). Correlation between Nutrition and Symptoms: Nutritional Survey of Children with Autism Spectrum Disorder in Chongqing, China. Nutrients, 8(5), 294. https://doi.org/10.3390/nu8050294.

McDaniel, J., Yoder, P., Woynaroski, T., \& Watson, L. R. (2018). Predicting Receptive-Expressive Vocabulary Discrepancies in Preschool Children With Autism Spectrum Disorder. Journal Of Speech Language And Hearing Research, 61(6), 1426-1439. https://doi.org/10.1044/2018_JSLHR-L-17-0101.

Mody, M., Shui, A. M. M., Nowinski, L. A. A., Golas, S. B. B., Ferrone, C., O’Rourke, J. A. A., \& McDougle, C. J. J. (2017). Communication Deficits and the Motor System: Exploring Patterns of Associations in Autism Spectrum Disorder (ASD). Journal of Autism and Developmental Disorders, 47(1), 155-162. https://doi.org/10.1007/s10803-016-2934-y.

Parmeggiani, A., Corinaldesi, A., \& Posar, A. (2019). Early features of autism spectrum disorder: a cross-sectional study. Ital J Pediatr, 45(1), 144. http://dx.doi.org/10.1186/s13052-019-0733-8. 
Research, Society and Development, v. 11, n. 1, e32111125015, 2022

(CC BY 4.0) | ISSN 2525-3409 | DOI: http://dx.doi.org/10.33448/rsd-v11i1.25015

Stevenson, J. L., Lindley, C. E., \& Murlo, N. (2017). Retrospectively Assessed Early Motor and Current Pragmatic Language Skills in Autistic and Neurotypical Children. Perceptual and Motor Skills, 124(4), 777-794. https://doi.org/10.1177/0031512517710379.

Thompson, A., Murphy, D., Dell'Acqua, F., Ecker, C., McAlonan, G., Howells, H., Baron-Cohen, S., Lai, M.-C. C., Lombardo, M. V., Dell’Acqua, F., Ecker, C., McAlonan, G., Howells, H., Baron-Cohen, S., Lai, M.-C. C., \& Lombardo, M. V. (2017). Impaired Communication Between the Motor and Somatosensory Homunculus Is Associated With Poor Manual Dexterity in Autism Spectrum Disorder. Biological Psychiatry, 81(3), 211-219. https://doi.org/10.1016/j.biopsych.2016.06.020.

Yang, S., Paynter, J. M., \& Gilmore, L. (2016). Vineland Adaptive Behavior Scales: II Profile of Young Children with Autism Spectrum Disorder. J Autism Dev Disord, 46(1), 64-73. http://dx.doi.org/10.1007/s10803-015-2543-1. 